tion of CO. The levels $v=2, J=13,14$ are near resonant with the most highly populated, $J=1-4,300 \mathrm{~K}$ Boltzmann states in $v=3$. V-R energy transfer from $v=3$ is, therefore, the only likely mechanism for the population of these levels:

$\mathrm{HF}(v=3, J=2)+\mathrm{CO}\left(v=0,\left\langle J_{i}\right\rangle\right) \rightarrow \mathrm{HF}(v=2, J=13)$

$$
+\operatorname{CO}\left(v=0,\left\langle J_{f}\right\rangle\right)+E \text { (Trans). }
$$

Table I shows the growth in the $(9 \leqslant J \leqslant 14)$ levels of $v=2$ together with the depletion of $v=3$ for the $\mathrm{F}+\mathrm{CH}_{4}$ reaction as $[\mathrm{CO}]$ is increased. Although the absolute emission intensity of the high $J$ levels in $v=2$ increases on addition of $\mathrm{CO}$, the increase is not linear indicating that some rotational quenching of these states into the lower $J$ Boltzmann envelope is occurring within the time scale of our observations. Even without allowing for partial relaxation of the initial high $J$ population formed in the quenching process, approximately $55 \%$ of the population loss from $v=3$ is channeled into the $J=9-14$ levels of $v=2$. Within our experimental uncertainty and accounting for the slight relaxation of $v=2$, the quenching loss from $v=3$ is equal to the total gain, summed over all $J$ states, in $v=2$. Together with our observation that levels $v=1, J \geqslant 15$ are not populated in the quenching process, the relaxation of $\operatorname{HF}(v=3)$ by $\mathrm{CO}$ proceeds mainly by processes of the form represented in Eq. (1).

Our figure of $55 \%$ constitutes a minimum value and indicates the importance of the near resonant $V-R, T$ channel in $\mathrm{CO}$ quenching of $\mathrm{HF}(v=3)$. Preliminary results indicate that $\mathrm{CO}$ quenching of $\mathrm{HF}(v=2)$ proceeds via a similar mechanism with the $\mathbf{V}-\mathrm{R}$ channel accounting for, at least, $70 \%$ of the total relaxation into $\operatorname{HF}(v=1)$. The preference for the V-R channel may arise from the strong intermolecular potential which favors the end-on, bonding of $\mathrm{H}$ to $\mathrm{CO}$, as observed in the linear OC-HF van der Waals complex. ${ }^{18}$ Quenching of $\mathrm{HF}(v)$ by $\mathrm{N}_{2}$ and $\mathrm{CO}_{2}$ also show significant $\mathrm{V}-\mathrm{R}, \mathrm{T}$ channels and for higher vibrational levels, additional near resonant $\Delta v=2$ relaxation channels become important.

\footnotetext{
a) Work supported by The National Science Foundation under Grant No. CHE-85-43609.

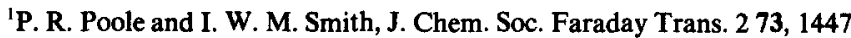
(1977).

${ }^{2}$ I. W. M. Smith and D. J. Wrigley, Chem. Phys. Lett. 70, 481 (1980).

${ }^{3}$ L. S. Dzelzkalns and F. Kaufman, J. Chem. Phys. 77, 3508 (1982).

${ }^{4}$ L. S. Dzelzkalns and F. Kaufman, J. Chem. Phys. 79, 3836 (1983).

${ }^{5}$ L. S. Dzelzkalns and F. Kaufman, J. Chem. Phys. 80, 6114 (1984).

${ }^{6}$ G. M. Jursich, D. R. Ritter, and F. F. Crim, J. Chem. Phys. 80, 4097 (1984).

7J. H. Smith and D. W. Robinson, J. Chem. Phys. 74, 5111 (1981).

${ }^{8}$ E. R. Sirkin and G. C. Pimentel, J. Chem. Phys. 75, 604 (1981).

${ }^{9}$ E. R. Sirkin and G. C. Pimentel, J. Chem. Phys. 77, 1314 (1982).

${ }^{10}$ G. L. Richmond and G. C. Pimentel, J. Chem. Phys. 80, 1162 (1984).

"X. Yang and G. C. Pimentel, J. Chem. Phys. 81, 1346 (1984).

${ }^{12}$ B. S. Agrawalla and D. W. Setser, in Gas Phase Chemiluminescence and Chemi-ionization, edited by A. Fontijn (Elsevier, New York, 1985).

${ }^{13}$ B. S. Agrawalla and D. W. Setser, J. Phys. Chem. 90, 2450 (1986).

${ }^{14}$ M. A. Wickramaaratchi, D. W. Setser, H. Hildebrandt, B. Korbitzer, and H. Heydtmann, Chem. Phys. 94, 109 (1985).

${ }^{15}$ J. F. Bott, J. Chem. Phys. 65, 4239 (1976).
}

${ }^{16}$ D. J. Douglas and C. B. Moore, J. Chem. Phys. 70, 1769 (1979).

${ }^{17}$ K. P. Huber and G. Herzberg, Constants of Diatomic Molecules (Van Nostrand Reinhold, New York, 1979).

${ }^{18}$ G. T. Fraser and A. S. Pine, J. Chem. Phys. 88, 4147 (1988).

\title{
Adsorption kinetics of surfactant molecules at a liquid-air interface
}

\author{
Th. Rasing, T. Stehlin, ${ }^{\text {a) }}$ and Y. R. Shen \\ Department of Physics, University of California, Center for Advanced Materials, Lawrence Berkeley \\ Laboratory, Berkeley, California 94720 \\ M. W. Kim and P. Valint, Jr. \\ Exxon Research and Engineering Company, Annandale, New Jersey 08801
}

(Received 20 April 1988; accepted 28 June 1988)

Surface active molecules, surfactants, are important for many practical applications such as lubrication, friction modifier, wetting control, and microelectronics. For these applications, one uses slightly soluble surfactants where the solubility is determined by the relative contributions of the hydrophilic and hydrophobic moeities. In those situations it is of interest to determine the kinetics of the monolayer formation from the bulk solution and the final composition of the surface layer. Traditionally, one uses surface tension and calorimetric measurements ${ }^{1}$ to investigate this adsorption kinetics. However, it is difficult to determine the surface concentration from these methods. ${ }^{2}$

In this paper we demonstrate the effectiveness of second harmonic generation technique (SHG) to obtain in situ both the adsorption kinetics and the final composition of a surfactant system. We also compare the result with surface tension measurements. The molecule under investigation is sodium-dodecylnapthalene-sulfonate

[SDNS; $\mathrm{CH}_{3}\left(\mathrm{CH}_{2}\right)_{11}-\mathrm{C}_{10} \mathrm{H}_{6}-\mathrm{SO}_{3} \mathrm{Na}$ ]. A saturated solution of SDNS in $2 \% \mathrm{NaCl}$ aqueous solution was prepared. A Langmuir trough, made out of glass with the edges wrapped with Teflon tape, was used to contain the solution. The surface tension was measured by a Wilhelmy plate.

The effectiveness of SHG as a surface probe has been demonstrated recently in a number of cases including liquid-air, ${ }^{3}$ liquid-liquid, ${ }^{4}$ and various other interfaces. ${ }^{5}$ As an 


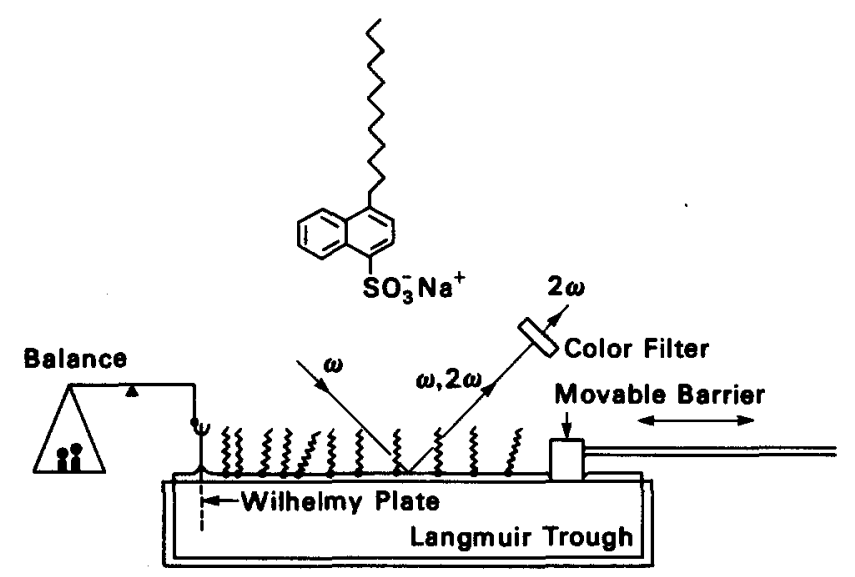

FIG. 1. Schematic diagram of experiments.

optical probe it has the advantage of a high spectral and time resolution, in situ and of being applicable to any interfaces accessible by light. It is based on the idea that the SHG is forbidden in centrosymmetric media but allowed at the interfaces where the inversion symmetry is necessarily broken. The surface nonlinear susceptibility $\chi_{s}^{2}$ which is responsible for the SHG at an interface generally reflects the properties of the surface layer. If $\chi_{s}^{2}$ arises mainly from a monolayer of surfactants, and takes the form

$$
\chi_{s}^{2}=N_{s}\left\langle\alpha^{2}\right\rangle,
$$

where $N_{s}$ is the surface density, and $\left\langle\alpha^{2}\right\rangle$ is the molecular nonlinear polarizability and the angular brackets \langle\rangle denote an average over all molecular orientations. In the previous publication, ${ }^{3}$ we have shown that the molecular orientation of SDNS on a water surface is only weakly dependent on the surface concentration. Therefore, if $N_{s}$ is varied, the SH signal should be directly proportional to the square of $N_{s}$. Thus, for the study of adsorption dynamics of SDNS, we can use SHG to moniter the change in surface density as a function of time.

The measurement of SDNS adsorption kinetics from the bulk solution to the air/solution interface was carried out by first cleaning the surface with Tefion barrier and the immediately proceeding with data taking. Figure 1 shows a schematic picture of the experiment and the structure of SDNS. Figure 2 shows the results of the SHG and surface tension measurements as a function of time. The dashed line is the theoretical fitting curve with Langmuir kinetics. If the adsorption barrier for the surfactant molecules is independent of the coverage $(\theta)$, then the change in $\theta$ is expected to followed Langmuir kinetics ${ }^{6}$ as

$$
\frac{d \theta}{d t}=K\left(\frac{1-\theta}{\theta_{0}}\right)
$$

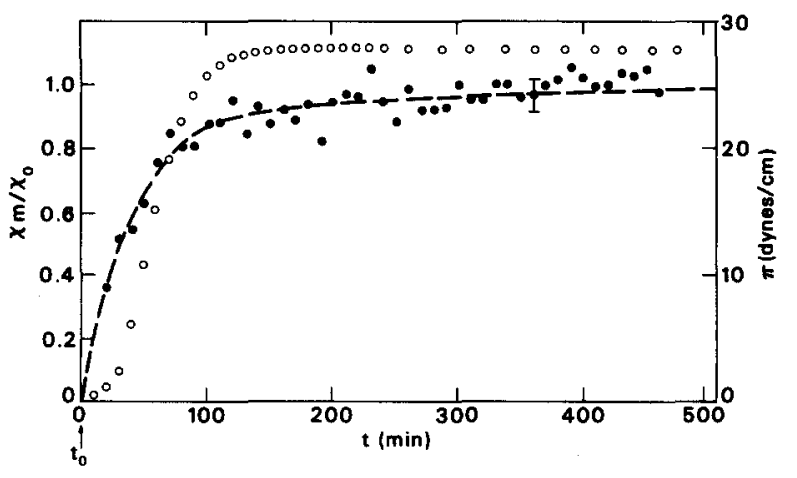

FIG. 2. Observed surface pressure $\pi$ (open circle) and nonlinear susceptibility $\chi$ for an air interface as a function of time after surface was SDNS saturated solution cleared. The dashed line is a Langmuir-type fit for the change in surface coverage.

where $\theta_{0}$ is the final coverage and $K$ a constant. From the SHG data, we can fit the time dependence of $\theta$ as

$$
\theta / \theta_{0}=1-a e^{-K t} .
$$

For $t<80 \mathrm{~min}$, we find $a=1.0$ and $1 / K=48 \mathrm{~min}$, while for $t>80 \mathrm{~min}$ we get $a=0.22$ and $1 / K=150 \mathrm{~min}$. For $t=\infty$, we find that $\theta_{0}=1$, i.e., the molecules eventually form a full monolayer, which is consistent with the surface tension measurements. The fact that there are two different time regime can be understandable in terms of the bulk surfactant diffusion control for an early stage adsorption and then the probability control of the creation of open area for new surfactant molecules for the late stage adsorption.

In conclusion, we have followed the kinetics of the surfactant adsorption from the bulk to the water-air interface by using the optical second harmonic generation. We have observed two different time regimes, the initial fast adsorption regime due to the surfactant bulk diffusion constant and the slow adsorption regime due to the sticking probability.

The part of this work was supported by the Director, Office of Energy Research, Office of Basic Energy Science, Materials Sciences Division of the U.S. Department of Energy under Contract No. DE-AC03-76SF00098.

\footnotetext{
a) Present address: Physikalisches Institut, Universitat Heidelberg, D-6900 Heidelberg 1, Federal Republic of Germany.

'D. K. Chattoraj and K. S. Birdi, Adsorption and the Gibbs Surface Excess (Plenum, New York, 1984).

${ }^{2} \mathrm{G}$. L. Gaines, Jr., Insoluble Monolayers at Liquid-Gas Interfaces (Wiley, New York, 1966).

${ }^{3}$ Th. Rasing, Y. R. Shen, M. W. Kim, P. Valint, Jr., and J. Bock, Phys. Rev. A 31, 537 (1985).

${ }^{4}$ S. G. Grubb, M. W. Kim, Th. Rasing, and Y. R. Shen, Langmuir 4, 452 (1988).

${ }^{5}$ Y. R. Shen, The Principle of Nonlinear Optics (Wiley, New York, 1984).

${ }^{6}$ B. B. Sauer, W. G. Griffin, and H. Yu, Macromolecules (to be published).
} 